\title{
Identification of Multi-Channel Simulated Auditory Event- Related Potentials using a Combination of Principal Component Analysis and Kalman Filtering
}

\author{
Kevin Paulson \\ School of Engineering and Computer Science \\ Hull, United Kingdom \\ K.Paulson@hull.ac.uk
}

\author{
Othman Alfahad \\ School of Engineering and Computer Science \\ Hull, United Kingdom \\ O.A.Alfahad@2014.hull.ac.uk
}

\begin{abstract}
The aim of this paper to develop a new method for separating auditory event-related potentials (ERP) signal from artefacts or noise. In experimental conditions, ERPs can be approximated by weighted sums of Principal Component Analysis (PCA) basis signals calculated from clean data. Projection of measured signals onto the PCA subspace significantly decreases noise. Furthermore, Kalman filtering has been used to optimize the combining of the PCA filtered signal with an a priori expected ERP. The main strength of the proposed algorithm arises from manipulating a priori cross-channel information in the form of a PCA weight covariance matrix. Here, the implementation of the method has been quantified using synthetic multi-channel ERP signals to which known amounts of synthetic noise is added to all the channels. The use of synthetic data means and signal and noise are known and so signal-to-noise enhancement may be quantified. For a wide range of initial SNRs, PCA filtering increases SNR by 10 $\mathrm{dB}$ and Kalman filtering yields an additional $10 \mathrm{~dB}$ improvement.
\end{abstract}

\section{CCS Concepts}

\begin{tabular}{|c|c|c|}
\hline $\begin{array}{l}\text { •Applied } \\
\text { engineering } \rightarrow\end{array}$ & $\begin{array}{l}\text { Computing } \rightarrow \text { Physical } \\
\text { Engineering. }\end{array}$ & sciences \\
\hline •Applied & Computing $\rightarrow$ Life & and \\
\hline
\end{tabular}

-General and reference $\rightarrow$ Cross-computing tools and techniques $\rightarrow$ Estimation

-Hardware $\rightarrow$ Emerging technologies $\rightarrow$ Biology-related information processing $\rightarrow$ Neural systems

\section{Keywords}

EEG; event-related potential; synthetic ERPs; PCA; Kaman filter; SNR; artifact removal.

\section{INTRODUCTION}

Auditory event-related potentials (ERPs) are measurements of

\footnotetext{
Permission to make digital or hard copies of all or part of this work for personal or classroom use is granted without fee provided that copies are not made or distributed for profit or commercial advantage and that copies bear this notice and the full citation on the first page. To copy otherwise, or republish, to post on servers or to redistribute to lists, requires prior specific permission and/or a fee. Request permissions from Permissions@acm.org.

ICBSP 2018, October 11-13, 2018, Bari, Italy.

(C) 2018 Association for Computing Machinery.

ACM ISBN 978-1-4503-6477-5/18/10 ..\$15.00

DOI: https://doi.org/10.1145/3288200.3288211
}

electroencephalographic (EEG) activity after an auditory stimulus. EEG recording commonly contains a variety of artefacts and noises. Significant difficulties and challenges in analysis and interpretation of EEG data to separate ERPs from other brain signals, non-neural artefacts such as muscle (EMG) and eye movements, artefacts due to equipment factors such as the movement of electrodes and wires, and interference from other systems [1]. Recording EEG signals from ambulatory or mobile EEG systems (MEEG) are usually contaminated by more and higher amplitude artefacts than static systems. However, researchers and clinicians wish to measure ERPs while individuals are involve in the physical activities associated with normal living [2]. Typically, single trial (epoch) ERP measurements are too noisy to allow ERPs to be detected. Noise artefacts may have amplitudes of tens or hundreds of micro-Volts $(\mu \mathrm{V})$ compared to ERP signals which typically have peaks of a few micro-Volts. Often, ERPs from many repeat measurements, on single or multiple individuals, are averaged before clinically important features can be observed. Coherent averaging is one technique to increase signal-to-noise ratio (SNR). Important features include the positive and negative voltage peaks, often labeled N100, P200, P300 etc. [3].

A standard multi channels EEG system provides 19 channels of differential ERP measurement from different standard locations on the scalp. Often, other channels record EOG, ECG, etc. [3], [4]. Each electrode measures the brain response, but filtered due to the electrode location and the intervening tissue, plus noise. This paper presents a method that allows averaging across channels. Although each channel measures a different view of the ERP response, a priori knowledge of the signal correlations allows the channels to be combined to yield a better approximation of the underlying ERP.

Ocular EEG artefacts, produced by eye movements and eye blinks, are often the most common and largest contaminating signals, particularly for electrodes near the front of the head. Often, epochs contaminated by EOG are eliminated from consideration. However, filtering out the EOG component of the measured EEG signal may leave more data for ERP identification. Principal Component Analysis (PCA) has been used to eliminate ocular artefacts in ERP signals by deducting the principal component related to eye artefacts for example, eye blinks, horizontal eye movements and vertical eye movements, [5] and [6]. Casarotto et al created an effectual and practical, PCA-based method for decreasing EOG artefacts [6]. Correspondingly, Kobayashi and Kuriki [7] applied PCA to increase the signal-to-noise ratio (SNR) in evoked neuromagnetic signals. Employing synthetic evoked fields they demonstrated SNR development and contrasted this with the common averaging method. 
Kalman filtering was developed by Kalman in 1960 for parameter estimation and has been broadly employed to parameter tracking applications in many fields. It yields the maximum likelihood estimator given a priori and posteriori estimates of parameter vectors, [8]. Kalman Filters (KF) have been applied to EEG time series. Kalman Smoothing has been used widely in EEG signals to detect spikes accompanying with psychological diseases, [9]. Oikonomou [10] noticed that there is a substantial improvement in EEG SNR when using time-varying coefficients for a signal autoregressive model, estimated using Kalman filtering.

This paper presents a method to estimate ERP responses from multi-channel EEG recordings. It is based on two stages. Firstly, ECG epochs are represented as a weighted sum of PCA basis vectors. Secondly, the PCA weights are refined using KF to find the optimal combination of measured and expected weights. In an earlier paper, [11], the authors present a similar method. That algorithm relied upon a PCA basis of signals for each channel of the EEG measurement. Much of the power of the method came from the a priori knowledge of the covariance between PCA basis signal weights across channels. For a 20 channel system, with 10 PCA basis signals for each channel, that method required the solution of matrix systems with rank 200. The method presented in this paper is a refinement of that algorithm. The signals measured on each channel are concatenated into a single ERP measurement vector. A collection of measurement vectors is used as training data and a PCA basis is calculated for the concatenated measurements. This method reduces the rank of the covariance matrix to 20 , and hence reduces the calculation involved by three orders of magnitude.

The paper begins by introducing a method to produce synthetic multi-channel ERP signals. These synthetic signals are used to quantify the performance of the proposed ERP estimation method. Section 3 describes the calculation of PCA bases for each channel ERP signal and the calculation of the covariance of channel PCA basis weights. The projection of ERP signals onto PCA bases, and the filtering it provides, is described in Section 4. Section 5 introduces Kalman filtering to provide the optimal combination of PCA filtered channel ERPs and the a priori expected multichannel ERP. Finally, Sections 6 test the algorithms with synthetic signals and noises and this is followed by a conclusions section.

\section{MULTI-CHANNEL ERP SIMULATION}

A method to produce synthetic, multichannel ERP signals has been presented in Paulson and Alfahad [11]. The multi-channel simulator assumes that all channels measure the same underlying brain process, but filtered by the bone and tissue between the activity and the electrode on the scalp. Therefore, many features of the ERP response will be consistent across channels. For example, the P300 response is assumed to be measured at the same time on all channels. Similarly, the response amplitude is assumed to vary proportionately across all channels. It is the resulting correlation across channels that will be exploited in the proposed algorithm introduced below.

The ERP signal on each channel is approximated by a sum of three Gaussian pulses, $N_{G}=3$ :

$$
\begin{gathered}
E_{i k}(t)=\sum_{j=1}^{N_{G}} A_{i j} f\left(t ; t_{j}, \sigma_{j}\right) \\
\text { where } f\left(t ; t_{j}, \sigma_{j}\right)=\exp \left(-\frac{1}{2 \sigma_{j}^{2}}\left(t-t_{j}\right)^{2}\right)
\end{gathered}
$$

$E_{i k}(t)$ is the pure ERP signal measured on the $i$ th channel, for the $k$ th trial. The three parameters for each pulse: $A_{i j}, t_{j}$, and $\sigma_{j}$; specify the amplitude, centre time and width of each of the Gaussian pulses respectively. For each trial, these parameters are determined from six independent samples from a Standard Normal distribution. Each trial is assumed to be independent [11].

\section{PRINCIPLE COMPONENT BASIS FOR ERP MEASUREMENTS}

An ERP measurement on a single channel yields a discrete voltage time series $\mathbf{E}_{i k} \in \mathrm{R}^{N_{s}}$, of $N_{s}$ samples. The measurement vector $\mathbf{E}_{k} \in \mathrm{R}^{N_{c} N_{s} \times 1}$ from the $k$ th trial can be formed from concatenating the ERP measurements from $N_{c}$ channels. If a set of $N_{t}$ ERP measurement vectors is available, then a mean vector and uncentred covariance matrix $\mathrm{C}_{\mathrm{E}} \in \mathrm{R}^{N_{c} N_{s} \times N_{c} N_{S}}$ may be estimated:

$$
\begin{aligned}
& \overline{\mathrm{E}}=\operatorname{mean}\left(\left\{\mathrm{E}_{k}\right\}, k=1, \cdots N_{t}\right) \\
& \mathrm{C}_{\mathrm{E}}=\operatorname{cov}\left(\left\{\mathrm{E}_{k}\right\}, k=1, \cdots N_{t}\right)
\end{aligned}
$$

The covariance matrix encapsulates all knowledge of the correlation between ERP samples on all channels. The mean state vector is our a priori best estimate of the multi-channel ERP measurement vector.

The Eigen vectors of the covariance matrix provide the principal component (PCA) basis signals. The Eigen values indicate how much of the variation between signals comes from components along PCA basis directions. Typically, the first few PCA basis signals span a large majority of the variation in ERP responses. Later basis signals span the noise. Projecting the ERP signal on the subspace spanned by the first few PCA basis signals will keep many of the features of the ERP signal while greatly reducing the noise. Let $\mathbf{B} \in \mathrm{R}^{N_{c} N_{s} \times N_{b}}$ be a matrix whose $N_{b}$ columns are the first $N_{b}$ PCA basis vectors of length $N_{c} N_{s}$ samples. The first PCA basis vector is the mean ERP signal: $\overline{\mathbf{E}}$. Projecting the measurement vector $\mathbf{E}_{k}$ onto the subspace spanned by the PCA basis vectors to get a vector of PCA basis weights $\mathrm{X}_{\boldsymbol{k}} \in \mathrm{R}^{N_{b}}$ can be written in matrix notation as:

$$
\mathbf{X}_{\boldsymbol{k}}=\mathbf{B}^{\mathrm{t}} \mathbf{E}_{\boldsymbol{k}}
$$

and the projected signal is:

$$
\mathbf{E}_{\text {pca }}=\mathbf{B X}_{k}=\mathbf{B B}^{\mathrm{t}} \mathbf{E}_{\boldsymbol{k}} \text {. }
$$

When the PCA weights are to be filtered, then a diagonal filter matrix $\mathbf{F} \in \mathrm{R}^{N_{b} \times N_{b}}$ may be introduced: $\mathbf{F} \equiv \operatorname{diag}\left(f_{i}\right)$. The filter weights can be chosen to yield a smooth truncation to avoid Gibbs ringing e.g. $f_{i}=\exp \left(-\left(i-T_{p c a}\right)^{2} / 2\right)$ where $T_{p c a}$ is the truncation basis number. The PCA filtered ERP signal may be written:

$$
\mathbf{E}_{\text {pca }}^{\mathbf{f}}=\mathrm{BFX}_{k}=\mathrm{BFB}^{\mathrm{t}} \mathbf{E}_{\boldsymbol{k}} \text {. }
$$

If $\mathbf{E} \in \mathrm{R}^{N_{c} N_{s} \times N_{t}}$ is a matrix whose $N_{t}$ columns are ERP measure vectors from $N_{t}$ trials, then (5) yields $\mathbf{E}_{\mathbf{p c a}}^{\mathbf{f}} \in \mathrm{R}^{N_{c} N_{s} \times N_{t}}$ a matrix of all-channel measurements after PCA filtering of each trial ERP measurement. A weighted sum of ERP measurements may be calculated by introducing a weight vector $\mathbf{W} \in \mathrm{R}^{N_{t}}$ where: $\mathbf{W} \equiv\left(w_{i}\right)$. The weighted mean ERP measurement may be written: $\overline{\mathbf{E}}=\mathbf{E W}$, and the PCA filtered mean measurement is:

$$
\overline{\mathbf{E}}_{\text {pca }}^{\mathbf{f}}=\text { BFB }^{\mathrm{t}} \overline{\mathbf{E}}=\text { BFB }^{\mathbf{t}} \mathbf{E W} \text {. }
$$

Due to the associative property of matrix multiplication, $\mathbf{B F B}^{\mathbf{t}}(\mathbf{E W})=\left(\mathbf{B F B}^{\mathbf{t}} \mathbf{E}\right) \mathbf{W}$, and so the weighted mean of the PCA 
filtered ERP response vectors is the same as the PCA filtered weighted mean ERP response.

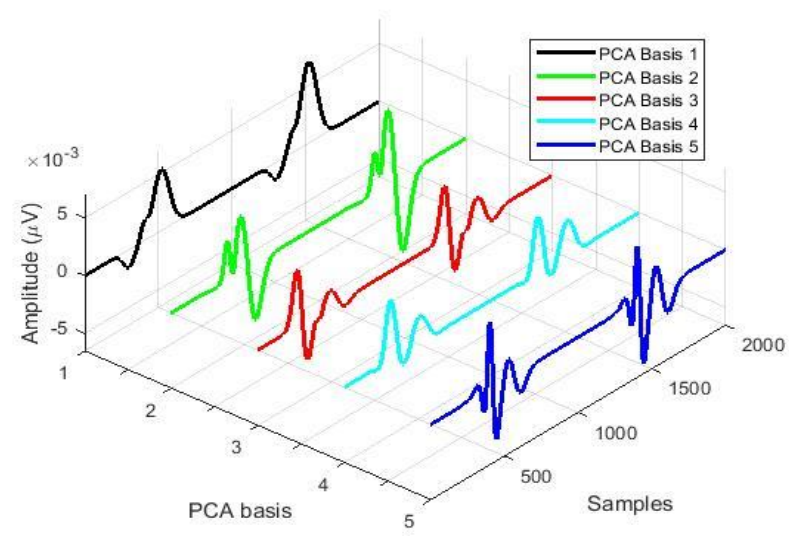

Figure 1. Sections of Principle Component Basis for first two channels

\subsection{Multi-Channel Information}

Using the multi-channel ERP simulator introduced in Section 2, the PCA basis matrix B can be calculated using the methods described in Section 3.1. As the underlying ERP signals have so much in common, only a small number of PCA basis vectors is required. For a given trial $k$, the filtered PCA projection weights can be calculated using:

$$
\mathbf{X}_{k}=\mathbf{F B}^{\mathrm{t}} \mathbf{E}_{k}
$$

Given a set of $N_{t}$ ERP PCA projected and filtered weights, then a mean vector $\overline{\mathbf{X}} \in \mathrm{R}^{N_{b} \times 1}$ and uncentred covariance matrix $\mathrm{C}_{\mathrm{X}} \in$ $\mathrm{R}^{N_{b} \times N_{b}}$ may be estimated:

$$
\begin{aligned}
& \bar{X}=\operatorname{mean}\left(\left\{X_{k}\right\}, k=1, \cdots N_{t}\right) \\
& C_{X}=\operatorname{cov}\left(\left\{X_{k}\right\}, k=1, \cdots N_{t}\right)
\end{aligned}
$$

The mean filtered weight vector is our a priori best estimate of the ERP PCA projected and filtered weight vector. The covariance matrix encapsulates all knowledge of the correlation between ERP filtered PCA weights.

\section{ERP ESTIMATION}

This section follows the conventional notation of Kalman Filtering, applied to an individual vector of filtered PCA weights i.e. an individual subject and trial. All the channels, as described by the vector $\mathbf{X}$, are estimated at the same time using an algorithm we call KFPCA. The estimation forms an optimal weighted sum of two sources of information: the a priori $\mathbf{X}$ and the measured $\mathbf{X}$.

A priori, an ERP state vector is a sample from the multidimensional Normal distribution with mean $\bar{X}$ and covariance $C_{X}$. In the absence of any further information, the maximum likelihood estimate of the ERP signal is that given by the mean vector $\bar{X}$. In terms of Kalman Filters the $\bar{X}$ and $C_{X}$ correspond to $\mathbf{X}_{k / k-1}$ and $\mathbf{P}_{k / k-1}$.

Given an ERP measurement on all channels, for a given subject and trial, a PCA projected and filtered weight vector $\widehat{\mathbf{X}}_{k}$ can be calculated using the processes in (3) and Section 3.1. The weights vector has an uncertainty covariance matrix $\mathbf{R}_{k}$ that is estimated from the difference between the reconstructed PCA filtered estimate $\mathbf{E}_{\mathrm{PCA}}^{\mathrm{f}}$ and the measurements $\mathbf{E}_{k}$, see Section 4.1. Given the a priori knowledge of ERP and the information provided by the measurement process, an estimate of the particular ERP for this subject and trial $\mathbf{X}_{k}$, can be calculated by solving (9) for the Kalman factor $\mathbf{K}$ and then using (10):

$$
\begin{gathered}
\mathbf{K}\left(\mathbf{C}_{\mathbf{X}}-\mathbf{R}_{\boldsymbol{k}}\right)=\mathbf{C}_{\mathbf{X}}, \\
\mathbf{X}_{k}=\widehat{\mathbf{X}}_{k}+\mathbf{K}\left(\widehat{\mathbf{X}}_{k}-\overline{\mathbf{X}}\right) .
\end{gathered}
$$

\subsection{Estimation of Measurement Uncertainty}

In order to implement Kalman Filtering it is necessary to develop an estimate of the measurement uncertainty covariance in a single vector of ERP filtered PCA weights. If $\mathbf{X}_{\mathbf{p c a}}^{\mathbf{f}}=\mathbf{F B}^{\mathbf{t}} \mathbf{E}_{k}$ are the filtered PCA measurement weights, then we need to estimate the measurement uncertainty matrix $\mathbf{R}_{k} \in \mathrm{R}^{N_{b} \times N_{b}}$ such that the probability density function for the actual ERP PCA weight vector, given the measurement, is multi-variate Normal with mean $\mathbf{X}_{\mathbf{p c a}}^{\mathbf{f}}$ and covariance $\mathbf{R}_{k}$. It will be assumed that the noise in the ERP measurement is uniformly distributed across the unfiltered PCA basis weights, and a full PCA basis of rank $N_{s}$. Let $n_{i}^{2}$ be the noise power or variance in the $i$ th PCA weight. Then, by Parseval's Theorem:

$$
N^{2}=\sum_{i=1}^{N_{s}} n_{i}^{2}
$$

where $N^{2}$ is the noise power in the measured ERP signal. This can be estimated by assuming the noise is close to the difference between the measured ERP signal and the PCA filtered signal:

$$
N^{2} \approx\left\|\mathrm{E}_{\mathrm{pca}}^{\mathrm{f}}-\mathrm{E}_{k}\right\|^{2} \text {. }
$$

Given (11) and the assumption of uniform distribution of noise, then for all $i$ :

$$
n_{i}^{2}=\frac{N^{2}}{N_{s}} \approx \frac{\left\|\mathrm{E}_{\mathrm{pca}}^{\mathrm{f}}-\mathrm{E}_{k}\right\|^{2}}{N_{s}}
$$

The covariance in the unfiltered PCA weights is then:

$$
\mathbf{R}_{\boldsymbol{k}}=\boldsymbol{n}_{\boldsymbol{i}}^{2} \mathbf{I}_{\boldsymbol{N}_{\boldsymbol{b}}} \text {. }
$$

where $\mathbf{I}_{N_{b}}$ is the identity matrix of rank $N_{b}$, and the covariance of the PCA filtered ERP is:

$$
\mathbf{R}_{k}=n_{i}^{2} \operatorname{diag}\left(w_{i}^{2}\right) .
$$

\section{SNR PERFORMANCE}

A simulated 20 channel MEEG system, with a $1 \mathrm{kHz}$ sample rate, has been used to test the performance of the PCA and KFPCA filters. The PCA basis, mean PCA weights vector $\overline{\mathbf{X}}$ and weights covariance matrix $\mathbf{C}_{\mathbf{X}}$ were calculated using 100 simulated multichannel ERP signals. Each channel signal is sampled from $200 \mathrm{~ms}$ before the stimulus to $800 \mathrm{~ms}$ after. In Monte Carlo tests, synthetic noise was added to the synthetic signals to simulate 100 ERP measurement trials for a range of noise scenarios. The ERP signal and synthetic noise was different for every trial. Three noise scenarios have been tested: additive white Gaussian noise (AWGN), additive pink Gaussian noise (APGN), and AWGN noise varying with channel. All noise was band limited to a maximum frequency of $15 \mathrm{~Hz}$ before SNR calculations. Signal and noise powers were defined to be the mean square amplitude of the 1000 sample signal sequences.

Figure 2 illustrates a single channel with band limited AWGN with an initial $\mathrm{SNR}=0 \mathrm{~dB}$. The black curve is the synthetic ERP signal produced by the multi-channel simulator, and for these tests can be taken as the true, noise-free ERP signal. The red curve is the measured signal including synthetic noise. PCA filtering 
reduces the noise in the signal by removing PCA components that are largely noise. In this example the PCA components are smoothly truncated around the 20th basis signal using Gaussian weights. This signal is further combined with the expected signal using a Kalman Factor to yield the green curve. Although only one channel is illustrated, the algorithm mixes information from all channels. All channels had the same initial SNR although, as the channel ERP signals have different amplitudes, the noise amplitudes will also change between channels. Figure 3 shows the SNR improvement with filtering, starting from a range of initial SNRs. PCA filtering (solid lines) yield about a $10 \mathrm{~dB}$ SNR improvement just by rejecting components that are predominantly noise. KFPCA (dashed lines) yields a further improvement of about $7 \mathrm{~dB}$ for relatively clean signals and the improvement grows linearly for noisy signals where the measured signal is largely disregarded and the a priori expected signal is returned as the most likely response. Note that some of the noise reduction comes from the projection method forcing the ERP signal to be zero before the stimulus and after the expected ERP response. A shorter time span, say from $-100 \mathrm{~ms}$ to $500 \mathrm{~ms}$, would yield a smaller SNR improvement.

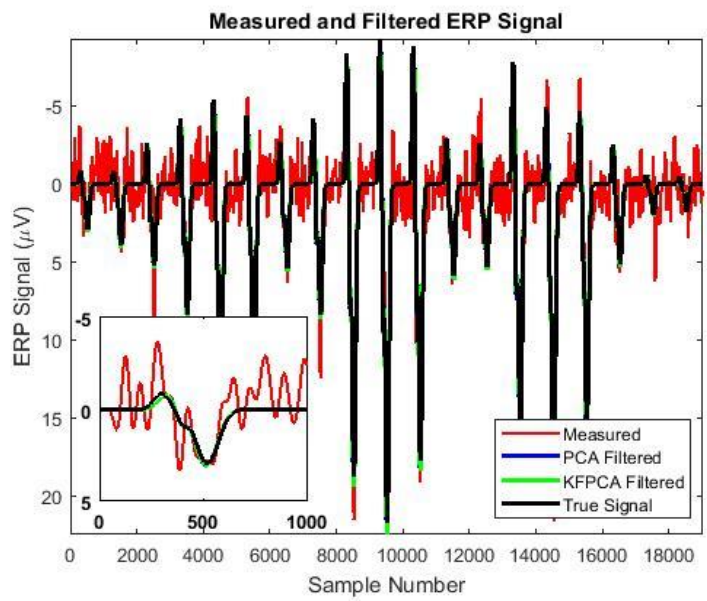

Figure 2. Measured, filtered and true ERP signal for band limited AWGN, SNR=0 dB. Inset is channel 1 signal.

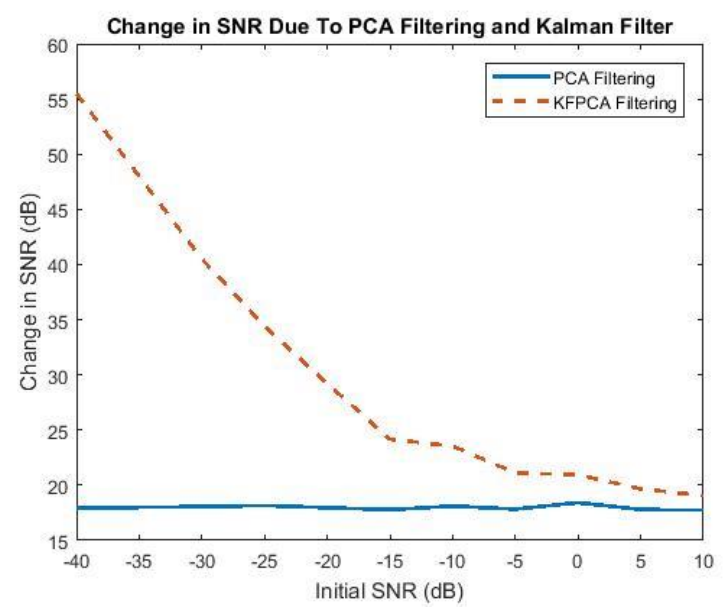

Figure 3. Change in SNR Due to PCA and Kalman Filtering for AWGN, for all channels

Figure 4 illustrates the results for the same experiment but using pink noise across all channels. Pink noise is much more challenging as it has more power at the lower frequencies which overlap more with the expected ERP signal. However, both PCA projection and Kalman filtering offer similar SNR improvements as with AWGN.

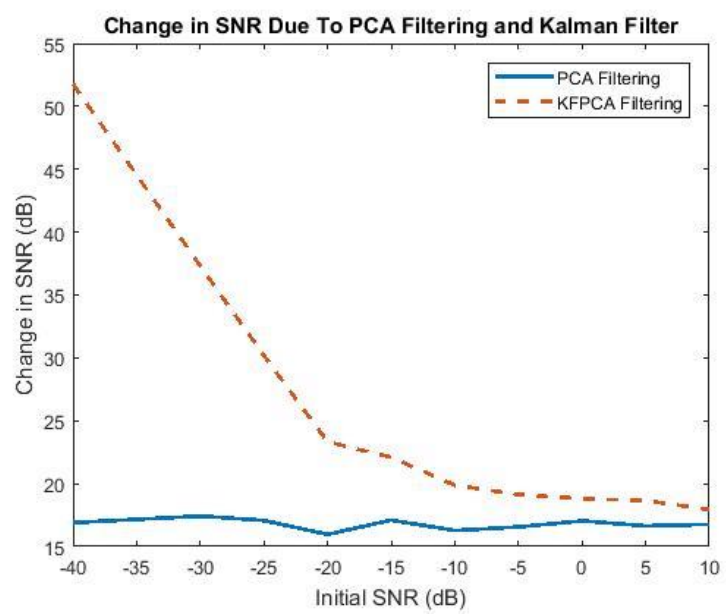

Figure 4. Change in SNR Due to PCA and Kalman Filtering for APGN.

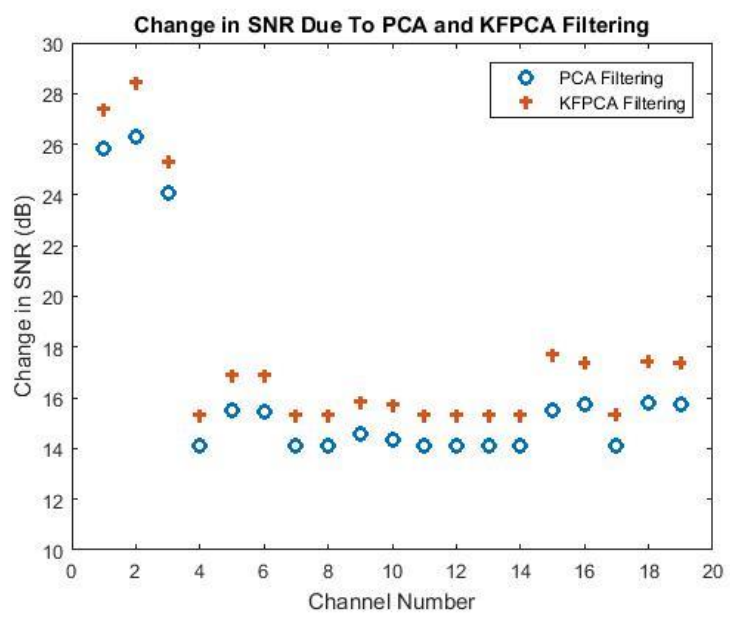

Figure 5. Change in SNR Due To PCA and Kalman Filtering where channels 1,2 and 3 have an initial $S N R=0$ while the other channels have an $S N R=10$.

Finally we consider the case where the initial SNR is not the same across all channels. This is typically the situation where noise is due to electrode connection problems, electrode movement or for biological signals such as eye blink artifacts. Eye blinks in particular effect the channels at the front of the head much more strongly than those further back. KFPCA filtering has the large advantage of utilizing expected correlations between all channel signals. Noisy channels are identified as part of the algorithm and the filtered signals on these channels are guided by the signals measured on the less noisy channels. This is illustrated in Figure 5 an example where channels 1, 2 and 3 (Fp1, Fp2 and F7) have initial $\mathrm{SNR}=0 \mathrm{~dB}$ while the other channels have $\mathrm{SNR}=10 \mathrm{~dB}$. For this test, AWGN was used. PCA filtering yields the same SNR improvement on all channels as the same noise amplitude exists in all the PCA bases. However, Kalman Filtering uses the crosschannel information and yields much higher SNR improvement in the three noisy channels. In effect the algorithm reconstructs the signals on these channels from the more reliable information measured on the relatively clean channels. 


\section{DISCUSSION AND CONCLUSION}

A synthetic multichannel ERP simulator has been used to demonstrate the SNR improvements produced by two denoising filter processes. Initially, a PCA basis is calculated for vectors formed by concatenations of channel measurements. In practice this would be done using a set of clean ERP signals derived from averages over many trials from many individuals. We have used synthetic ERP signals as these allow SNR improvements to be calculated. PCA filtering reduces artefact noise by projecting measured ERP signals onto low dimensional subspaces spanned by the first 20 principal component signals in each channel. A smooth truncation was used to reduce Gibbs ringing. In these tests, PCA projection increased SNR by about $10 \mathrm{~dB}$ for both white and pink Gaussian noise. In a second stage, the PCA projection weights were optimally combined with a priori weights using knowledge of the weights covariance and a Kalman factor. In effect, this uses information from all channels to reduce noise in each channel. The method was demonstrated in a scenario where three channels initially had $10 \mathrm{~dB}$ more noise than the others, to simulate eye blink artifacts. After KFPCA filtering, all channels had near the same SNR. This method provides a new way to interpolate missing channels that is much more sophisticated than methods based on weighted sums of adjacent channels. We propose to use KFPCA filtering on real data as a preprocessor, before combining trial outputs in a statistically optimised way to estimate ERP signals in the minimum number of trials.

\section{ACKNOWLEDGMENT}

This work was supported in part by the Saudi Arabia, Prince Sultan Military College of health sciences under Grant MD207.

\section{REFERENCES}

[1] T. Jung et al, "Removing electroencephalographic artifacts by blind source separation," Psychophysiology, vol. 37, (2), pp. 163-178, 2000.
[2] A. D. Bateson et al, "Categorisation of Mobile EEG: A Researcher's Perspective," BioMed Research International, vol. 2017, 2017.

[3] S. J. Luck, "An introduction to the event-related potential technique MIT press," Cambridge, Ma, pp. 45-64, 2005.

[4] S. Debener et al, "How about taking a low-cost, small, and wireless EEG for a walk?" Psychophysiology, vol. 49, (11), pp. 1617-1621, 2012.

[5] J. C. Costa et al, "Validation in principal components analysis applied to EEG data," Comput. Math. Methods Med., vol. 2014, pp. 413801, 2014.

[6] S. Casarotto et al, "Principal component analysis for reduction of ocular artefacts in event-related potentials of normal and dyslexic children," Clin. Neurophysiol., vol. 115, (3), pp. 609-619, Mar, 2004.

[7] T. Kobayashi and S. Kuriki, "Principal component elimination method for the improvement of $\mathrm{S} / \mathrm{N}$ in evoked neuromagnetic field measurements," IEEE Transactions on Biomedical Engineering, vol. 46, (8), pp. 951-958, 1999.

[8] R. E. Kalman, "A new approach to linear filtering and prediction problems," Journal of Basic Engineering, vol. 82, (1), pp. 35-45, 1960.

[9] V. P. Oikonomou et al, The use of Kalman Filter in Biomedical Signal Processing. INTECH Open Access Publisher, 2009.

[10] V. P. Oikonomou, A. T. Tzallas and D. I. Fotiadis, "A Kalman filter based methodology for EEG spike enhancement," Comput. Methods Programs Biomed., vol. 85, (2), pp. 101-108, 2007.

[11] K. S. Paulson and O. A. Alfahad, "Estimation of auditory event-related potentials using a combination of principal component analysis and kalman filtering," in Proceedings of the 4 thWorld Congress on Electrical Engineering and Computer Systems and Science (EECSS'18), Madrid, 2018. 